\title{
Editorial
}

\section{Zukunftsdialog KI und Bildung}

https://doi.org/10.1515/iwp-2019-2070

„KI macht Schule“ war das Motto des DGI-Forums Wittenberg 2019. Im Wissenschaftsjahr 2019, das dem Thema „Künstliche Intelligenz“ gewidmet war, wollte die Deutsche Gesellschaft für Information und Wissen gemeinsam mit dem Bereich Deutschdidaktik der Martin-Luther-Universität Halle-Wittenberg mit ihrer Tagung den Wirkungen von Digitalisierung und KI im Bildungssektor auf die Spur kommen und den Zukunftsdialog darüber, der bereits bei den DGI-Praxistagen 2018 begonnen hatte, fortsetzen. Die anregende Tagung bot zahlreiche Denkanstöße und zeigte erwartete und unerwartete Parallelen zum Bibliotheksund Informationsbereich.

Die Digitalisierung von Dokumenten und Medien aller Art, die Internettechnologie zur weltweiten Vernetzung und die allgemein zugänglichen unterschiedlichsten Kommunikationsdienste haben zu tiefgreifenden Veränderungen bei Informationsgewinnung, Nachrichtenverbreitung und Wissenserwerb geführt.

Bibliotheken im traditionellen Sinn als Hort von Büchern und Medien, den man persönlich aufsucht, um sich etwa mit den Gedanken von Autoren auseinanderzusetzen, um sich Wissen anzueignen, um sich an Lyrik zu erbauen, sich mit Reiseschriftstellern in fremde Welten zu begeben oder in eine Welt der Fiktion einzutauchen, sind heute weitgehend der „Bibliothek als drittem Ort“ gewichen. Sie sind ein Treffpunkt, ein angenehm klimatisierter und ansprechend ausgestatteter Aufenthaltsort, der unentgeltlich elektrische Energie zum Aufladen der mitgeführten elektronischen Geräte und jedwede Infrastruktur zur Verfügung stellt, einschließlich sachkundigen, hilfsbereiten Dienstleistern - derzeit noch echtem menschlichem Personal und der meistens mit einem eigenen kommunikationsfähigen Endgerät - oder auch gleich mehreren - aufgesucht wird.

Könnte sich auch die Schule zu einem „dritten Ort“ wandeln, zumal wir einen Trend zur Ganztagsschule haben und die Kinder immer mehr Zeit in der Schule verbringen? Weitgehend einig sind sich Pädagogen und die aufgeklärte Öffentlichkeit, dass Bildung weit über das Erlernen von Rechnen und Schreiben sowie die Kenntnis faktischer jederzeit online abrufbarer Informationen hinausgeht. Bildung ist ohne ein Mindestmaß an Wissen zwischen den Ohren zwar nicht möglich, Urteilsfähigkeit und Handlungskompetenz im gesellschaftlichen und poli- tischen Umfeld müssen aber hinzukommen. Mit dem Digitalpakt werden fünf Milliarden Euro in den nächsten fünf Jahren für die kommunikationstechnische Ausstattung von Schulen in Deutschland bereitgestellt, doch damit wird lediglich eine technische Infrastruktur verfügbar. Genauso wichtig sind Medien für den Unterricht und das Selbststudium, die das Verstehen und Lernen anregen und unterstützen, sowie didaktische Konzepte, die Wege durch den Dschungel der digitalen Informationen und Angebote bahnen und die Kinder und Jugendlichen auf die digitalisierte Welt vorbereiten. Bedienkompetenz ihrer Geräte und der darauf installierten Anwendungen bringen Schülerinnen und Schüler meistens mit, der Abruf von Alltagsinformationen oder Gebrauchsanleitungen und der flinke Dialog miteinander sind Standard. Was also muss Schule als Bildungsort leisten?

In einem Bildungsort Schule könnte das individuelle Einüben von Fertigkeiten wie etwa Rechnen, Schreiben, Vokabellernen mit Software unterstützt werden, möglicherweise auch durch eine KI, die aufgrund einer Fehleranalyse erkennt, wo Übungsbedarf oder Wissenslücken bestehen und passende Erklärungen liefert oder Aufgaben zum Entdecken neuer Lösungswege stellt. Hierzu könnten neben Aufenthaltsbereichen auch Einzel- und Gruppenarbeitsplätze ähnlich denen in Lernzentren der Hochschulen dienen.

Emotionale und soziale Bildung dürfte hingegen auch künftig durch menschliche Begegnungen und gemeinsame Aktivitäten im Klassenverband oder in Lerngruppen entwickelt werden. Hierzu wird die Schule weiterhin Begegnungsort sein, um gemeinsam Sport zu treiben, zu musizieren, zu debattieren, zu philosophieren, vorzutragen, Theater zu spielen, sich künstlerisch und kreativ mit Themen auseinander zu setzen. Auch Klassenfahrten oder Exkursionen werden wohl kaum durch VR-Erlebnisse ersetzt werden. Trotzdem muss die Schule selbstverständlich mit digitalen und KI-Anwendungen vertraut machen und diese didaktisch sinnvoll einsetzen, um den aufgeklärten Umgang damit zu vermitteln.

Angesichts des Lehrkräftemangels ist es wenig zielführend, die Lehrkräfte zusätzlich mit Administrationsaufgaben für die IT-Infrastruktur zu betrauen. Eher müssen einfach zu bedienende und lokal beherrschbare technische Lösungen genutzt werden, die die informationelle Selbstbestimmung und Unabhängigkeit von werbefinanzierten Konzernen sicherstellen. Ob in jedem einzelnen Fall 
Cloud-basierte Dienste oder Online-Angebote genutzt werden müssen oder auch Intranet-Angebote und schulinterne Server ausreichen, müssen die Schulgremien als demokratisch gewählte Organe der Schule aushandeln.

Hilfreich könnte eine KI eventuell zur Entlastung der Lehrkräfte von Verwaltungsaufgaben sein, Einsatzplanung, Raumplanung, Anmeldeverfahren, Stundenplan aufstellen oder auch bei der Kommunikation mit Eltern in anderen Sprachen.

Die vorliegende Ausgabe der IWP enthält die Textfassungen einiger Beiträge des DGI-Forums Wittenberg 2019 und eine von Matthias Ballod und Stefanie Klein durchgeführte erste Auswertung der Online-Umfrage zu KI und Bildung, die im Vorfeld durchgeführt worden war.

Olivetta Gentilin zeigt, wie sich computerlinguistische Anwendungen beim Fremdsprachenunterricht einsetzen und zur Textanalyse nutzen lassen. Jürgen Buder stellt die Frage, unter welchen Bedingungen ein Dialogsystem oder ein Roboter als menschenähnlich empfunden wird. Jörn Loviscach bezweifelt, dass die Phänomene Wissbegier und Lernen überhaupt schon verstanden, geschweige denn durch Bildungstechnologien gefördert oder angeregt werden, und fragt, ob Motivationsproblemen nicht in Wirklichkeit mit sozialpolitischen, gesellschaftlichen und ökonomischen Lösungsansätzen begegnet werden muss.
Inga Kampmann, Pablo Pirnay-Dummer und Inka Hähnlein stellen eine Lösung für eine neuartige, epistemologische Domänenmodellierung vor, die als Alternative zu ontologischen, hierarchischen Ansätzen angewendet werden kann. Die Wissensdomänen können von Institutionen wie Universitäten und Bibliotheken als Instrument zum innovativen Wissensmanagement eingesetzt werden. Ziel dabei ist es, eine adäquate Re-Repräsentation menschlichen Wissens bereitzustellen, mit der Menschen auf natürliche Weise interagieren können, um zu lernen und ihr Wissen zu erweitern.

Sue-Ann Bäsler und Felix Sasaki erläutern wie ein Schulbuchverlag den digitalen Wandel umsetzt und vor allem mit Methoden des maschinellen Lernens Lernplattformen sowie Bildungsmedien entwickelt.

Bettina Gierke stellt Zielsetzung und Stand des Fachinformationsdienstes Buch-, Bibliotheks- und Informationswissenschaft vor, der den Spezialbedarf der Wissenschaft in den genannten und einigen angrenzenden Disziplinen decken soll.

Wir wünschen allen Leserinnen und Lesern eine anregende Lektüre und freuen uns über ihre Reaktionen. Das Thema wird in der DGI-Fachgruppe Informationskompetenz und Bildung weiterverfolgt. Sie sind zur Teilnahme herzlich eingeladen. 